\title{
Current advances in animal model of chondrosarcoma and related research (Review)
}

\author{
YU-XIN LIAO $^{1}$, YING-QI HUA ${ }^{1}$ and ZHENG-DONG CAI ${ }^{2}$ \\ ${ }^{1}$ Musculoskeletal Oncology Center; ${ }^{2}$ Department of Orthopaedics, Shanghai Tenth People's Hospital, \\ Tongji University School of Medicine, Shanghai 200072, P.R. China
}

Received July 30, 2012; Accepted September 17, 2012

DOI: $10.3892 /$ br.2012.13

\begin{abstract}
Chondrosarcoma is a type of malignant cartilage tumor with a high local recurrence. Due to its resistance to chemo- and radiotherapy, current treatment is limited to surgical resection. Animal model is one of the most important approaches to studying this disease, although systematic reporting on its development is rare. In this review, we summarized the elements involving animal model establishment. On the basis of these elements, we further classified chondrosarcoma animal models into various types. In addition, we compared various measurements for evaluating the animal model. Finally, its specific applications were discussed.
\end{abstract}

\section{Contents}

1. Introduction

2. Elements of chondrosarcoma animal model

3. Experimental animals

4. Cell lines for transplantation

5. Graft sites

6. Classification of chondrosarcoma animal model

7. Application of chondrosarcoma animal model

8. Conclusions

\section{Introduction}

Chondrosarcoma is the second most common primary malignant bone tumor that predominantly occurs in adults $>40$ years of age $(1,2)$. Due to its poor response to chemo-, as well as radiotherapy, at present surgical resection is an effective treatment for chondrosarcoma $(3,4)$. This mesenchymal

Correspondence to: Professor Zheng-Dong Cai, Department of Orthopaedics, Shanghai Tenth People's Hospital, Tongji University School of Medicine, 301 Yanchang Road, Shanghai 200072, P.R. China

E-mail: zhdcai@gmail.com

Key words: chondrosarcoma, animal model, allograft, xenograft, heterotopic, orthotopic malignancy has a poor prognosis with local recurrence and a 5-year survival rates of 24-33 and 64-77\%, respectively $(5,6)$. The predilection sites of chondrosarcomas are pelvic bones and femur, nevertheless, the majority of tumors grow slowly. Although metastasis is infrequent, the lung is the most common site of metastasis in chondrosarcoma (7-9).

\section{Elements of chondrosarcoma animal model}

The experimental animal model that closely resembles human chondrosarcoma in clinic is considered to be one of the most important approaches to studying tumor growth, progression and metastasis. Three key factors including experimental animal, cell lines for transplantation and graft sites should be taken into account when building the chondrosarcoma animal model.

\section{Experimental animals}

Mice and rats are considered to be optimal choices in animal experiments. There are many different strains that we can utilize. Sprague-Dawley rats have been used as implanted animal models for a long time. In their study 45 years ago, Maibenco et al (10) reported that an 18 month-old female Sprague-Dawley rat (SD rat) developed a spontaneous chondro-blastic-osteogenic tumor in the thoracic and lumbar vertebrae. The tumor was resected surgically to establish a chondrosarcoma cell line designated as Swarm rat chondrosarcoma (SRC) for transplantation (11). Varying types of rats deliver varying results to a certain extent. Mason and Bansal (12) demonstrated that the tumor weight in Lewis rats was two times bigger compared to Wistar rats at the same time point. Due to lack of host immune response, nude mice, such as Balb/c nude mice and severe combined immune deficiency (SCID) mice have been increasingly used by scientific researchers in recent years. Human chondrosarcoma cells can thus be implanted without host immune rejection.

\section{Cell lines for transplantation}

Cell lines for graft are mainly composed of animal and human chondrosarcoma cell lines, according to different sources (13). The animal chondrosarcoma cell lines primarily refer to SRC derived from a female SD rat with spontaneous tumor, as 
mentioned previously (11). SRC-JWS and SRC-TRO cell lines are mostly used in animal-derived chondrosarcoma cell lines. Stevens (11) compared the differences in subcutaneous tumor growth using these two cell lines. The first palpable tumor can be detected 7 days earlier in the SRC-JWS compared to the SRC-TRO group. Tumors ( 35 and $11 \mathrm{~g}$ ) were harvested on days 21 and 35, for the SRC-JWS and SRC-TRO cell lines, respectively (11). The SRC-JWS cell line exhibited a more rapid growth and aggressive behavior compared to SRC-TRO. Similar to the experimental model selection, selection of cell line also influences tumor growth in animal models. A number of additional human chondrosarcoma cell lines are also being investigated, including OUMS-27, HCS-2/A, HCS-TG, NCDS-1, SW1353, JJ012, FS090 $(1,13)$. To the best of our knowledge, apart from being two unique human chondrosarcoma cell lines for orthotopic xenograft models, JJ012 and SW1353 are the most frequently applied in human chondrosarcoma transplantation.

\section{Graft sites}

Regardless of the types of chondrosarcoma cell lines chosen, subcutaneous and tibia are the two most common locations for implantation. Due to the easy application, subcutaneous injection had been widely adopted over the past decades, especially with SRC cell lines. With the deepening of cognition, more attention is paid to the implantation of tumor cells into tibia, where chondrosarcomas often occur. In addition, in their study, Clark et al (1) made a comparison of two transplantation sites using intratibial and periosteal injections. Their findings demonstrated that more time was required to reach the same tumor size in the intratibial compared to the periosteal group. They argued that this discrepancy may be due to the fact that vascular supply was more direct through the adjacent femoral vessels by the periosteal compared to the intratibial injection (1). Additionally, blood supply has been generally considered to be involved in tumorigenesis (14).

\section{Classification of the chondrosarcoma animal model}

Allograft and xenograft models. Transplantation animal models can be divided into allograft and xenograft models, depending on the origin of the chondrosarcoma cell lines used. The process that implants animal-derived cells, such as SRC, in most cases into animal, is termed allograft. Similarly, xenograft refers to the process whereby human chondrosarcoma cell are injected into animals. SD rats as usual experimental animals have been utilized in allograft models for a long time. When using this model, tumor growth and lung metastases were only $2 / 9$ and $1 / 24$, separately $(15,16)$. Due to lack of relevance to human disease, allograft models are now infrequently employed. Although this model has several weaknesses, in their study, Clark et al (13) suggested allograft models to be useful for the evaluation of chondrosarcoma growth and histology rather than the development of novel therapeutic agents. The use of nude mice made xenograft models possible. Without host rejection response, human chondrosarcoma cells can be implanted. Clark et al (1) injected JJ012 cells into the intramedullary canal of the left tibia and the periosteum of the anterior tibia in female, 5-week-old Balb/c nude mice. Their findings demonstrated that the average of implanted tumors by periosteal injection was $174.2 \mathrm{~mm}^{3}$ at 7 weeks, and $198.5 \mathrm{~mm}^{3}$, by intratibial at 10 weeks. Seven weeks after implantation, lung metastases rates were $5 / 5$ and 2/4, respectively, which is markedly better compared to the allograft model. In their study, Klenke et al (17) injected SW1353 cells subcutaneously into the left flank of SCID mice to assess the inhibitory effect of tyrosine kinase inhibitor SU6668 on chondrosarcoma growth in vivo.

Heterotopic and orthotopic models. Depending on the graft sites, transplantation animal models comprise heterotopic and orthotopic models. Subcutaneous implantation is the most common method in heterotopic models. SRC, the animal-derived chondrosarcoma cell, has mostly been used as a subcutaneously implanted graft (13). Orthotopic transplantation mainly refers to the injecting of chondrosarcoma cells into the marrow cavity of the tibia, where primary chondrosarcoma often occurs. Transplantation sites have been shown to affect the malignancy of the tumor i.e., the grade of the tumor formed by orthotopic transplantation is higher compared to that of a tumor formed by heterotopic transplantation (16). In their study, Hamm et al (18) verified the varying effect of SRC on tumor growth and metastasis at different transplantation sites. Their findings suggested that after 3 weeks the average weight of tumors induced by heterotopic and orthotopic transplantations was $35.05 \mathrm{~g}$ and $75.22 \mathrm{mg}$, respectively. Lung metastasis can be detected in $50 \%$ of the orthotopic group animals, whereas in none in the heterotopic group animals. Although the former induced obviously larger tumors compared to the latter, the orthotopic transplantation resulted in more aggressive tumors that were capable of invading the surrounding bone tissue (16). The tumors exhibited various growth and histologic characteristics, depending on transplantation sites (18). This is the reason that a growing number of investigators prefer to build orthotopic models (Fig. 1).

Evaluation of chondrosarcoma animal models. Tumor formation and lung metastasis rates are two important indicators when assessing chondrosarcoma animal models. Implantation tumors growth condition can be evaluated by tumor weight and volume in most animal experimental studies. Tumor weight can be easily measured by scales subsequent to resection, while the traditional method to measure tumor volume is using a caliper. Its volume value can be calculated by the formula: $\mathrm{V}=0.5 \times \mathrm{LxS}^{2}$, where $\mathrm{L}$ and $\mathrm{S}$ stand for the largest and smallest perpendicular tumor diameters, respectively (19). Whether or not lung metastasis occurs is mainly assessed by hematoxylin and eosin (H\&E) staining and immunohistochemistry of the lung tissue harvested from sacrificed animals. Accumulating evidence has demonstrated that bioluminescent imaging (BLI) is a promising technique used widely in the field of pre-clinical oncology research (20). It provides rapid, non-invasive monitoring of tumor growth and regression in animals, quantitative and sensitive analysis of tumor growth and metastasis, assessment of tumor development and responses to drug therapies in vivo (21). In their study, Comstock et al (22) compared BLI and the caliper approach for the measurement of primary tumor volume in nude mice. Their findings showed that there was a good linear correlation 


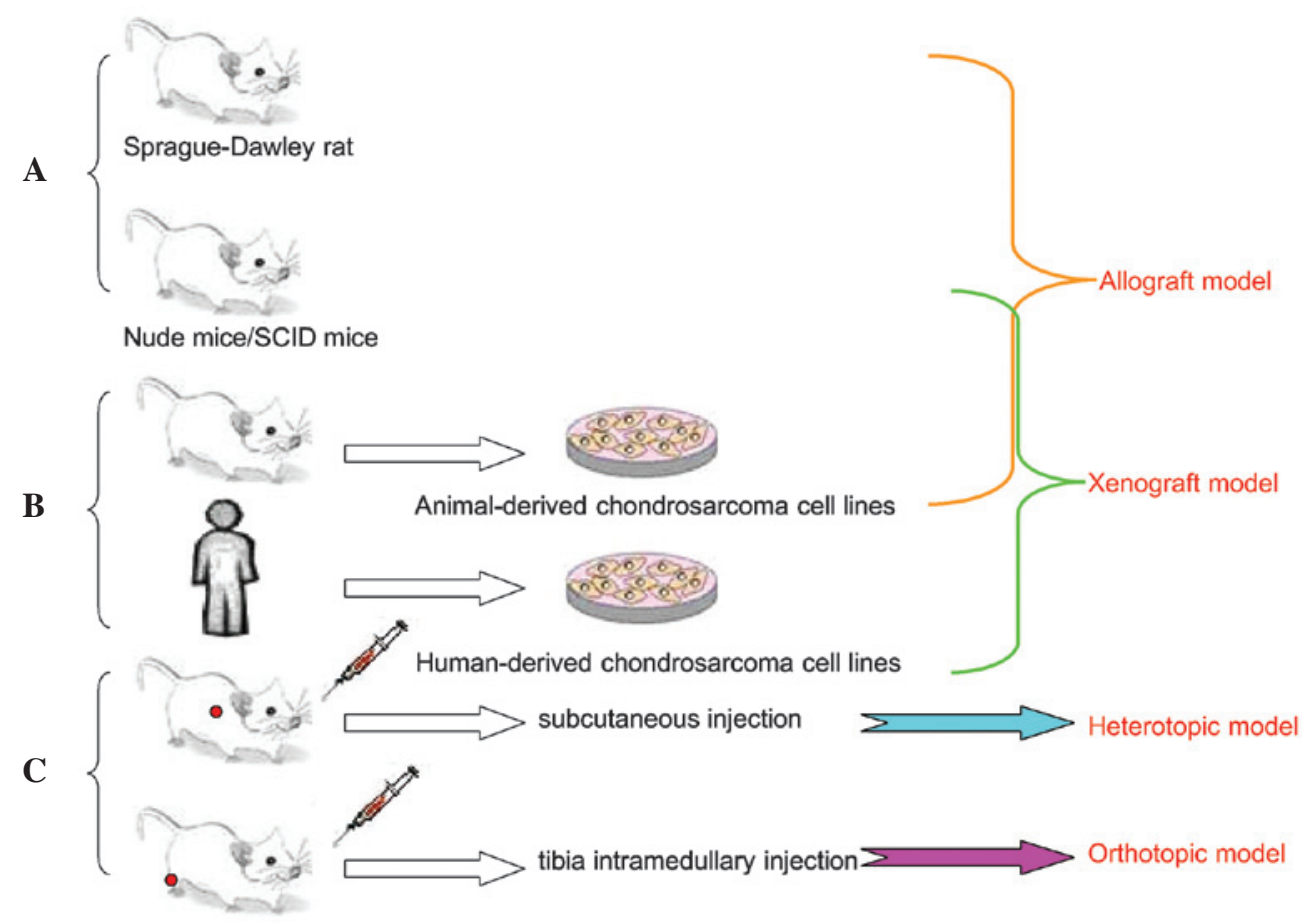

Figure 1. Elements and classification of the chondrosarcoma animal model are shown. (A) Experimental animals involved in chondrosarcoma animal model establishment mainly comprise Sprague-Dawley rat and nude mice/severe combined immune deficiency (SCID) mice. (B) Cell lines for transplantation are mostly divided into animal- and human-derived chondrosarcoma cell lines. (C) Graft sites primarily consist of subcutaneous and tibia intramedullary injection. Allograft model is established by injecting animal-derived chondrosarcoma cell lines into Sprague-Dawley rats. The xenograft model is built by injecting human-derived chondrosarcoma cell lines into nude or SCID mice. Heterotopic and orthotopic models are constructed by subcutaneous and tibia intramedullary injection, respectively.

between these two methods, while the tumors can be detected an average of 12.5 days earlier, when using BLI compared to caliper measurement (22). Consistent with their study, similar conclusions were drawn by Honigman et al (23) suggesting that malignant cells be visualized using BLI at least 13 days prior to palpable tumor formation in certain xenograft models. The advantages of BLI over the traditional approaches are: it permits real-time monitoring of tumor growth, spread, response to treatment in pre-clinical cancer models using the same animal without sacrifice $(24,25)$, and the detection of unexpected micrometastases that are frequently too small to be visible and thus may be neglected using traditional methods (21). The prerequisite of BLI is the establishment of the chondrosarcoma cell lines, which can stably express luciferase by transfection. Factors affecting the overall sensitivity of BLI include signal depth, transgene expression level and the extent of background bioluminescence (20). Bioluminescent light emission attenuates with the increased tissue depth. Besides, the change in tumor shape during growth and tumor necrosis at later time points are also responsible for imprecise measurement when using BLI (22).

\section{Application of the chondrosarcoma animal model}

The chondrosarcoma animal models are mainly used to investigate novel effective therapies to treat this disease. Gouin et al (19) determined the effects of zoledronic acid (ZOL) on chondrosarcoma tumor progression. They constructed an allograft model of SD rats by placing a $10 \mathrm{~mm}^{3}$
SRC fragment. Four days subsequent to implantation, the rats were randomized into two groups: the ZOL-treated and control groups. In the ZOL-treated group each rat received $100 \mu \mathrm{g} / \mathrm{kg}$ ZOL subcutaneously twice a week. The average tumor volume in the ZOL-treated group was demonstrated to be markedly smaller compared to the control group on day $25\left(4318 \pm 2278\right.$ and $10355 \pm 7414 \mathrm{~mm}^{3}$, respectively) and on day $27\left(5253 \pm 4133\right.$ and $15092 \pm 10781 \mathrm{~mm}^{3}$, respectively). Survival rate in ZOL-treated group was $0.667 \pm 0.33$ compared with $0.3 \pm 0.197$ in the control group on day 40 . Liu et al (26) developed a locoregional recurrence model of chondrosarcoma by injecting CS-1 cells, an aggressive human chondrosarcoma cell line, into the dorsum of 6 to 8 -week-old female NU/J mice to evaluate the effect of paclitaxel-eluting polymer film on reducing locoregional recurrence rates and improving survival rates. Those authors did not perform an R0/R1 resection until the volume of primary tumors reached approximately $500 \mathrm{~mm}^{3}$. The mice were then randomized into four groups: implantation of Pax-film containing $300 \mu \mathrm{g}$ paclitaxel, implantation of unloaded film, intravenous (IV) injection of $300 \mu \mathrm{g}$ paclitaxel in Cremophor/ethanol and no additional treatment. Locoregional recurrence was observed in 2 of 12 Pax-film mice (17\%), 9 of 13 unloaded-film mice (69\%), 8 of 9 Pax IV mice (89\%), and 7 of 8 untreated mice $(88 \%)$ within 100 days. The median overall survival was 81 , 64, 48 and 56 days, respectively. Their findings indicated that continuous local drug release by polymer films was a potential novel approach for the treatment of local aggressive chondrosarcoma (26). 
In addition to discovering new remedies, chondrosarcoma animal models were utilized for other studies. In their study, Yonekawa et al (27) established an SRC animal model to investigate whether or not serum cartilage-derived retinoic-acid-sensitive protein (CD-RAP) could be used as a marker of tumor activity. Their findings demonstrated that there was a positive correlation between serum CD-RAP level and tumor growth. A marked decrease in the serum CD-RAP level was observed subsequent to tumor resection, which increased prior to tumor recurrence.

\section{Conclusions}

Chondrosarcoma is a primary malignant bone tumor that responds poorly to chemo- as well as radiotherapy. Despite adopting surgical resection, the therapeutic effect remains unsatisfactory and has high local recurrence and low 5-year survival rates. Management of this disease remains an ongoing challenge. The animal model, which closely mimics human chondrosarcoma in clinic, is considered to be an indispensable tool when exploring the pathogenesis, metastasis and drug resistance mechanism of chondrosarcoma or evaluating the therapeutic effect of novel treatments. Although as discussed previously, several types of chondrosarcoma animal models are available, orthotopic xenograft models remain the gold standard in oncology research. With the wide application of BLI, the animal model that can be used for in vivo imaging facilitates the non-invasive, rapid and precise detection of tumor growth.

\section{References}

1. Clark JC, Akiyama T, Dass CR and Choong PF: New clinically relevant, orthotopic mouse models of human chondrosarcoma with spontaneous metastasis. Cancer Cell Int 10: 20, 2010.

2. Hemmati M, Abbaspour A, Alizadeh AM, et al: Rat xenograft chondrosarcoma development by human tissue fragment. Exp Oncol 33: 52-54, 2011.

3. Li TM, Lin TY, Hsu SF, et al: The novel benzimidazole derivative, MPTB, induces cell apoptosis in human chondrosarcoma cells. Mol Carcinog 50: 791-803, 2011.

4. Bergh P, Gunterberg B, Meis-Kindblom JM and Kindblom LG: Prognostic factors and outcome of pelvic, sacral, and spinal chondrosarcomas: a center-based study of 69 cases. Cancer 91: 1201-1212, 2001.

5. Fiorenza F, Abudu A, Grimer RJ, et al: Risk factors for survival and local control in chondrosarcoma of bone. J Bone Joint Surg Br 84: 93-99, 2002.

6. Bruns J, Elbracht M and Niggemeyer O: Chondrosarcoma of bone: an oncological and functional follow-up study. Ann Oncol 12: 859-864, 2001.

7. Qureshi A, Ahmad Z, Azam M and Idrees R: Epidemiological data for common bone sarcomas. Asian Pac J Cancer Prev 11: 393-395, 2010.
8. Gelderblom H, Hogendoorn PC, Dijkstra SD, et al: The clinical approach towards chondrosarcoma. Oncologist 13: 320-329, 2008.

9. Ozaki T, Hillmann A, Lindner N, Blasius S and Winkelmann W: Metastasis of chondrosarcoma. J Cancer Res Clin Oncol 122: 625-628, 1996

10. Maibenco HC, Krehbiel RH and Nelson D: Transplantable osteogenic tumor in the rat. Cancer Res 27: 362-366, 1967.

11. Stevens JW: Heterogeneity in growth properties of the rat Swarm chondrosarcoma. Iowa Orthop J 24: 33-35, 2004.

12. Mason RM and Bansal MK: Different growth rates of swarm chondrosarcoma in Lewis and Wistar rats correlate with different thyroid hormone levels. Connect Tissue Res 16: 177-185, 1987.

13. Clark JC, Dass CR and Choong PF: Development of chondrosarcoma animal models for assessment of adjuvant therapy. ANZ J Surg 79: 327-336, 2009.

14. Folkman J: The role of angiogenesis in tumor growth. Semin Cancer Biol 3: 65-71, 1992.

15. Kenan S and Steiner GC: Experimental transplantation of the Swarm rat chondrosarcoma into bone: radiological and pathological studies. J Orthop Res 9: 445-451, 1991.

16. Grimaud E, Damiens C, Rousselle AV, Passuti N, Heymann D and Gouin F: Bone remodelling and tumour grade modifications induced by interactions between bone and swarm rat chondrosarcoma. Histol Histopathol 17: 1103-1111, 2002.

17. Klenke FM, Abdollahi A, Bertl E, et al: Tyrosine kinase inhibitor SU6668 represses chondrosarcoma growth via antiangiogenesis in vivo. BMC Cancer 7: 49, 2007.

18. Hamm CA, Stevens JW, Xie H, et al: Microenvironment alters epigenetic and gene expression profiles in Swarm rat chondrosarcoma tumors. BMC Cancer 10: 471, 2010.

19. Gouin F, Ory B, Redini F and Heymann D: Zoledronic acid slows down rat primary chondrosarcoma development, recurrent tumor progression after intralesional curretage and increases overall survival. Int J Cancer 119: 980-984, 2006.

20. O'Neill K, Lyons SK, Gallagher WM, Curran KM and Byrne AT: Bioluminescent imaging: a critical tool in pre-clinical oncology research. J Pathol 220: 317-327, 2010.

21. Jenkins DE, Oei Y, Hornig YS, et al: Bioluminescent imaging (BLI) to improve and refine traditional murine models of tumor growth and metastasis. Clin Exp Metastasis 20: 733-744, 2003.

22. Comstock KE, Hall CL, Daignault S, Mandlebaum SA, Yu C and Keller ET: A bioluminescent orthotopic mouse model of human osteosarcoma that allows sensitive and rapid evaluation of new therapeutic agents In vivo. In Vivo 23: 661-668, 2009.

23. Honigman A, Zeira E, Ohana $\mathrm{P}$, et al: Imaging transgene expression in live animals. Mol Ther 4: 239-249, 2001.

24. Sarraf-Yazdi S, Mi J, Dewhirst MW and Clary BM: Use of in vivo bioluminescence imaging to predict hepatic tumor burden in mice. J Surg Res 120: 249-255, 2004.

25. Doyle TC, Burns SM and Contag $\mathrm{CH}$ : In vivo bioluminescence imaging for integrated studies of infection. Cell Microbiol 6: 303-317, 2004

26. Liu R, Wolinsky JB, Catalano PJ, et al: Paclitaxel-eluting polymer film reduces locoregional recurrence and improves survival in a recurrent sarcoma model: a novel investigational therapy. Ann Surg Oncol 19: 199-206, 2012.

27. Yonekawa M, Kondo S, Sugiura H, et al: Serum cartilage-derived retinoic acid-sensitive protein (CD-RAP) levels in swarm rat chondrosarcoma. J Orthop Res 20: 382-386, 2002. 